\title{
LEADER FOLLOWER FORMATION CONTROL FOR UNDERWATER TRANSPORTATION USING MULTIPLE AUTONOMOUS UNDERWATER VEHICLES
}

\author{
F U Rehman, E Anderlini and G Thomas, University College London, UK
}

\section{SUMMARY}

The successful ability to conduct underwater transportation using multiple autonomous underwater vehicles (AUVs) is important for the commercial sector to undertake precise underwater installations on large modules, whilst for the military sector it has the added advantage of improved secrecy for clandestine operations. The technical requirements are the stability of the payload and internal collision avoidance while keeping track of the desired trajectory considering the underwater effects. Here, a leader-follower formation control strategy was developed and implemented on the transportation system of AUVs. PID controllers were used for the vehicles and a linear feedback controller for maintaining the formation. A Kalman Filter (KF) was designed to estimate the full state of the leader under disturbance, noise and limited sensor readings. The results demonstrate that though the technical requirements are met, the thrust oscillations under disturbance and noise produce the undesired heading angles.

\section{NOMENCLATURE}

$\boldsymbol{B}$

$\mathrm{BFF}$

$\boldsymbol{C}_{\boldsymbol{R B}}(\boldsymbol{v})$

$C_{A}(v)$

C

$D_{L}(v)$

$D_{Q}(v)$

$\mathrm{EFF}$

$e_{\lambda x}, e_{\lambda y}$

$e_{\psi}$

f

$\boldsymbol{g}(\boldsymbol{\eta})$

$I_{z}\left(l_{x}, l_{y}, l_{z}\right)$

J

$\mathrm{EFFK}_{\boldsymbol{f}}$

$K_{P}$

$K_{i}$

$K_{d}$

k

$M_{R B}$

$M_{A}$

$m$

$\mathrm{O}$

Pose

RMS

RMSE

$T_{a}$

$\boldsymbol{u}$

uDist

uNoise

$\left(u_{L}, v_{L}, r_{L}\right)$

$\left(u_{F}, v_{F}, r_{F}\right)$

$(u, v, r)$

$\boldsymbol{V}_{\boldsymbol{d}}$

$V_{n}$

$v$
State matrix

Control input matrix

Body-fixed frame

Rigid Body Coriolis matrix

Added mass Coriolis matrix

Output matrix

Linear damping matrix

Quadratic damping matrix

Earth-fixed frame

Error between desired and actual

distances between leader and follower

Error of heading angle between leader and follower

Thrust force vector

Vector of hydrostatic forces and moments

Moment of inertia about z-axis

Position of thruster

Transformation matrix from BFF to

Kalman filter gain

Proportional gain

Integral gain

Differential gain

Formation control gain

Rigid body mass matrix

Added mass matrix

Mass of HAUV

Centre of origin

Position and Orientation

Root Mean Square

Root Mean Square Error

Thrust allocation matrix

Control input vector

Random disturbance

Random noise

Velocities of the leader in BFF

Velocities of follower in BFF

(surge velocity, sway velocity, yaw rate)

Disturbance covariance

Noise covariance

Velocity vector in BFF

$\begin{array}{ll}\boldsymbol{v}(\boldsymbol{t}) & \text { Noise vector } \\ \boldsymbol{v}_{\boldsymbol{r}} & \text { Relative velocity in BFF } \\ \boldsymbol{v}_{\boldsymbol{c}} & \text { Sea current velocity in BFF } \\ \boldsymbol{w}(\boldsymbol{t}) & \text { Disturbance vector } \\ \left(X_{\dot{u}}, Y_{\dot{v}}, N_{\dot{r}}\right) & \text { Added mass in surge, sway and yaw } \\ \left(X_{u}, Y_{v}, N_{r}\right) & \text { Linear damping in surge, sway and yaw } \\ \left(X_{u|u|}, Y_{v|v|}, N_{r|r|}\right) & \text { Quadratic damping in surge, sway } \\ & \text { and heave } \\ \boldsymbol{x} & \text { State vector } \\ \widehat{\boldsymbol{x}} & \text { Estimated state vector } \\ (x, y, \psi) & \text { (surge, sway, yaw) } \\ \left(x_{L}, y_{L}, \psi_{L}\right) & \text { Pose of leader } \\ \left(x_{F}, y_{F}, \psi_{F}\right) & \text { Pose of follower } \\ \left(x_{F d}, y_{F d}\right) & \text { Desired position of the follower } \\ \boldsymbol{y} & \text { Vector of sensor readings } \\ \boldsymbol{\eta} & \text { Pose vector } \\ \dot{\boldsymbol{\eta}} & \text { Velocity vector in EFF } \\ \boldsymbol{\eta}_{\boldsymbol{d}} & \text { Desired pose vector } \\ \lambda & \text { Distance from leader to follower in EFF } \\ \left(\lambda_{x}, \lambda_{y}\right) & \text { Components of } \lambda \\ \boldsymbol{\tau} & \text { Thrust vector } \\ \boldsymbol{\tau}_{\boldsymbol{d}} & \text { Desired thrust vector } \\ \psi_{L F} & \text { Heading angle difference between leader } \\ & \text { and follower in EFF }\end{array}$

\section{INTRODUCTION}

Unmanned underwater vehicles (UUVs) are increasingly used worldwide for underwater applications such as ship's underwater hull maintenance, hydrographical surveys and mineral field surveys. UUVs can undergo tasks that are difficult or risky for humans (Wang et al., 2009). UUVs are also used in military applications; in fact, they were first developed for the US Navy for underwater searching (Wernli, 2000). However, other sectors soon realised their potential, such as the oil and gas industry (Williams, 2004).

There are mainly two types of underwater vehicles: Remotely Operated Vehicles (ROVs) and Autonomous Underwater Vehicles (AUVs). ROVs are tethered and are generally over-actuated i.e. more thrusters than the degrees of freedom which helps them move in any direction precisely. The major drawback of a ROV is the high drag force due to its box shape. The drag force is prominent at high speeds. Therefore, ROVs are recommended to be 
operated at slow speeds (Frost \& Mcmaster, 1996). On the other hand, AUVs are programmed to accomplish a predefined task (Blidberg, 2010). They are underactuated and can be operated efficiently at high speeds due to their shape. Therefore, these vehicles are used in applications where a large area is required to be searched (Miller et al., 2014). For underwater transportation, underwater vehicles need to be autonomous and precise in operation to ensure the stability of the payload. This can be achieved by operating ROV autonomously and are named the Hovering Autonomous Underwater Vehicles (HAUVs).

Payload transportation is an important requirement for various sectors, which is generally fulfilled by land, aerial and sea surface vehicles. Underwater transportation is not commonly used due to increased hull resistance (Rehman et al., 2019). Nevertheless, the use of subsea transportation can be inevitable in certain circumstances such as in the military sector where the military payload is required to be transported undetected. Moreover, it can also be used in the offshore industry, especially to install underwater structures or rigs with precision. However, it is only recommended for short-range operations.

The major advantages of using multiple vehicles are flexibility in using the number of vehicles based on the mass of the payload and fault tolerance (Ghommam et al., 2010).

Underwater transportation analysis has the following challenges.

1. The dynamics of the UUV in the underwater environment is highly nonlinear and coupled. This increases the complexity of the development of a simulation model. Any assumption regarding the linearisation or decoupling would result in the deviation of the results from the actual.

2. The hydrodynamic parameters could be calculated either numerically, empirically or by model testing. All these methods result in uncertainties since the parameters are calculated in certain conditions as the exact underwater scenario cannot be predicted. Therefore, the dynamic model which contains the hydrodynamic parameters also has uncertainties that affect the motion response and stability of the system.

3. The underwater environment has severe communication constraints. Many research groups are investigating solutions to improve underwater communication such as by using optic sensors along with ultrasonic sensors (Farr, 2018) (Lloret et al., 2012). However, these technologies are not yet commercially available. Therefore, only ultrasonic sources can be used, which have underwater limitations such as low bit rates, dropouts, and delays (Millan et al., 2014).

The three main types of formation control strategies are Virtual-Structure, Behaviour-Based and Leader-Follower (LF).

In the virtual-structure formation control strategy, the entire formation is treated as a single entity. The desired motion is assigned to the virtual structure which traces out the trajectories for each member of the formation to follow (Lawton et al., 2003). Due to the consideration of a single virtual rigid structure, this strategy can result in a common point of failure for the entire system (Mehrjerdi et al., 2011). To counter this problem, a Control Lyapunov Function was defined for each vehicle in the group in reference (Ogren \& Egerstedt, 2002) so that the motion control problem of the multiple agents is converted into a stabilization problem of a single system.

In the behaviour-based formation control strategy, several desired behaviours are prescribed for each vehicle in the group. The final control is derived from a weighting of the relative importance of each behaviour. The controller of each behaviour for each vehicle activates when the situation appears. The virtual structure and leader-follower formation control strategies require that the full state of the leader is communicated to each member of the formation. However, the behaviour-based approach is decentralised and can be implemented with significantly less communication (Lawton et al., 2003). However, this method is mathematically difficult to analyse due to the inclusion of several behaviours for each vehicle and the relative switching of the behaviours by the other members in the group. Also, this switching and relative changes between the behaviours of vehicles makes it hard to guarantee a precise formation control or formation keeping (Lawton et al., 2003)(Mehrjerdi et al., 2011). Moreover, this method does not guarantee the stability of the whole system (Qi, 2014).

In the LF strategy, one of the autonomous vehicles in the group is designated as the leader and the rest as the followers. The followers need to maintain a desired position relative to the leader (Mehrjerdi et al., 2011). For stability, each vehicle in the formation must have a stable tracking control (Dai et al., 2010).

A wide range of research has been accomplished to develop a LF control strategy for multiple autonomous vehicles. For instance, in (Mercado-ravell et al., 2013), it was developed for the flight formation control of quadrotors. The sliding mode controller was used to get the desired orientation for the leader's quadrotor which was controlled by the PD controller. Furthermore, the sliding mode controller was applied on the error dynamics of the formation to get the desired states of the follower which was then controlled by the sliding mode controller. Whereas in (Wu et al., 2017), both the leader and follower were controlled using PID controllers and a sliding mode controller was used for maintaining the formation. In (Wang et al., 2009), a LF formation strategy was developed for Autonomous Underwater Vehicles using linear feedback controller on the formation dynamics to get the desired distance and angle between the leader and follower.

Though LF is a simple formation control strategy, the formation keeping is not guaranteed because there is no feedback. Therefore, if a follower fails to follow properly, no mechanism can guarantee the formation keeping (Yu et al., 2017). In general, this strategy depends on the leader vehicle. Therefore, if the leader vehicle experiences a 
malfunction, the whole system would be paralysed (Qi, 2014).

For multi-vehicular transportation, the LF strategy can be implemented in two possible ways. Firstly, as used in reference (Yufka \& Ozkan, 2015) for autonomous land vehicles, the payload is assumed to be the virtual leader and all the vehicles are taken as followers. In the other approach, which was adopted in (Yang et al., 2004), one of the vehicles is assumed to be a leader and others the followers.

In this paper, the LF strategy is used for underwater transportation with multiple autonomous underwater vehicles using the strategy in which one of the vehicles is considered as a leader and rest as the followers. This solution seems practically more attractive, as in practice the sensors are on the vehicles, so the leader tracks the trajectory and the followers sense the position of the leader.

The rest of the paper is organised as follows. Section 2 discusses the development of a dynamic model for HAUV. Section 3 describes the LF formation control strategy. Section 4 describes the transportation system on which the developed strategy is required to be implemented. A trajectory is generated for the leader in Section 5. In Section 6 , the results are discussed after implementing the developed strategy on the transportation system and finally concluding remarks are given in Section 7.

\section{DEVELOPMENT OF DYNAMIC MODEL FOR HAUV}

The dynamic model is developed to get the mathematical representation of the actual vehicle. The motion of a UUV is highly nonlinear and coupled, therefore a dynamic model is required to account for it.

In this paper, a dynamic model was developed for the Minerva HAUV (the name given as the Minerva ROV was used autonomously). This is a standard vehicle which is operated by the Norwegian University of Science and Technology (NTNU) (Lysdahl, 2010) and the model parameters are readily available in the NTNU's papers, such as reference (Mo, 2015). The Centre of Gravity (COG) is the point at which the resultant weight of the vehicle is applied. Whereas, on Centre of Buoyancy (COB), the resultant buoyancy force is applied. For static stability, COG should be below COB. For dynamic stability, certain criteria are required to be met. The Minerva HAUV has both static and dynamic stability. It is operated at slow speeds due to its box shape. Moreover, it is over actuated therefore it can move precisely in any direction. This property is of great advantage for multi-vehicular transportation as it ensures the stability of the payload.

Two reference frames were used in the dynamic model. The position and orientation were defined in the Earth-Fixed Frame (EFF), whereas, the velocities, forces and moments were expressed in the Body-Fixed Frame (BFF).

\section{$2.1 \quad$ ASSUMPTIONS}

The following assumptions were made in the development of the dynamic model for a Minerva HAUV:
1. The vehicle is a rigid body of constant mass. This ensures that there is no relative motion between the mass particles of the system (Peraire and Widnall, 2008). Also, mass and its distribution do not change which ensures that the COG does not change during the task.

2. The vehicle is deeply submerged; therefore, wave effects are ignored.

3. The Coriolis terms are ignored due to short-range operation.

4. The hydrostatic terms are ignored due to analysis in the horizontal plane in which they are less significant.

5. The interaction effects with other bodies are ignored.

6. The vehicle was assumed to be neutrally buoyant.

\subsection{EQUATIONS OF MOTION}

The equations of motion for Minerva HAUV consist of kinematics and kinetics. The kinematics describes the motion of the vehicle without taking into account the forces and moments that cause the motion (Beggs, 1983). The forces and moments are covered in the kinetics. The internal terms of kinetics are hydrodynamics and hydrostatics. The hydrodynamics consist of added mass and drag terms which depend on the accelerations and velocities. The hydrostatic terms are worked out from the difference between weight and buoyancy of the vehicle. The angular components of hydrostatics depend on the rotations. On the other hand, the external terms of kinetics of HAUV are the thrust forces and moments which are controlled to get the desired motion response. In this paper, the motion response is analysed in the horizontal plane.

After transformation into EFF, the kinematic equations in the horizontal plane are given as

$$
\begin{gathered}
\dot{x}=u \cos \psi-v \sin \psi, \\
\dot{y}=u \sin \psi+v \cos \psi, \\
\dot{\psi}=r .
\end{gathered}
$$

These can also be written in the vectorial form, given as

$$
\dot{\eta}=J v \text {. }
$$

where

$$
\dot{\boldsymbol{\eta}}=\left[\begin{array}{c}
\dot{x} \\
\dot{y}
\end{array}\right], \quad \boldsymbol{J}=\left[\begin{array}{ccc}
\cos \psi & -\sin \psi & 0 \\
\sin \psi & \cos \psi & 0 \\
0 & 0 & 1
\end{array}\right], \quad \boldsymbol{v}=\left[\begin{array}{c}
u \\
v \\
r
\end{array}\right] .
$$

On the other hand, the kinetic equations for a seagoing vessel either on the surface or underwater is derived from the Newton-Euler formulation. For instance, Fossen derived the equations of motion in 6DOF taking into consideration all the nonlinearities and couplings (Fossen, 2011).

The kinetics equations were derived from the first principles in this research. The Centre of Origin (CO) is the bodyfixed reference point. It was assumed that $\mathrm{CO}$ is coinciding COG for the HAUV. Newton's second law was expressed based on momentum theory which is divided into Euler's 
first and second axioms. The first Euler's axiom helps derive the translational terms of rigid body equations of motion and the second axiom derives the rotational terms. After derivation, the kinetic equations can be written in the matrices form as

$\left(M_{R B}+M_{A}\right) \dot{v}+\left(C_{R B}(v)+C_{A}(v)\right) v+D_{L}(v) v$

$+D_{Q}(v) v|v|+g(\eta)=\tau$

Considering the assumptions given in Section 2.1, equation (5) is reduced to the following

$$
\left(M_{R B}+M_{A}\right) \dot{v}+D_{L}(v) v+D_{Q}(v) v|v|=\tau
$$

Moreover, due to two planes of symmetry in the horizontal plane of the Minerva HAUV, the matrices in equation (5) can be written in the reduced form as

$$
\begin{gathered}
\boldsymbol{M}_{\boldsymbol{R B}}=\left[m, m, I_{z}\right]^{D} . \\
\boldsymbol{M}_{\boldsymbol{A}}=\left[X_{\dot{u}}, Y_{\dot{v}}, N_{\dot{r}}\right]^{D} . \\
\boldsymbol{D}_{\boldsymbol{L}}(\boldsymbol{v})=\left[X_{u}, Y_{v}, N_{r}\right]^{D} . \\
\boldsymbol{D}_{\boldsymbol{Q}}(\boldsymbol{v})=\left[X_{u|u|}, Y_{v|v|}, N_{r|r|}\right]^{D} .
\end{gathered}
$$

All the parameters in (5) for a Minerva HAUV were obtained from reference (Mo, 2015) which are based on DNV-GL standards (DNV, 2010).

Equation (5) can also be written in the state dynamics form as

$$
\dot{v}=\left(M_{R B}+M_{A}\right)^{-1}\left(-D_{L}(v) v-D_{Q}(v) v|v|+\tau\right) .
$$

Combining the kinetics and kinematics state dynamics from equations (4) and (11), the complete state dynamics of the vehicle is written as

$$
\dot{\boldsymbol{x}}=\left[\begin{array}{c}
\dot{\boldsymbol{\eta}} \\
\dot{\boldsymbol{v}}
\end{array}\right]
$$

where $\dot{\boldsymbol{\eta}}=\left[\begin{array}{c}\dot{x} \\ \dot{y} \\ \dot{\psi}\end{array}\right]$ and $\dot{\boldsymbol{v}}=\left[\begin{array}{c}\dot{u} \\ \dot{v} \\ \dot{r}\end{array}\right]$.

The $4^{\text {th }}$ order Runge-Kutta method was used for the time integration to simulate the motion response of the HAUV.

\subsection{PROPULSION MODEL}

The propulsion model determines the thrust forces and moments required to control the motion response of the vehicle. Five thrusters are installed on the Minerva HAUV i.e. two axial, two vertical and one transverse, as shown in Figure 1 . The effect of each thruster is required to be taken about CO.

In this paper, the thrusters are controlled by their thrust forces. The thrust vector is written as a product of thrust allocation matrix $\left(\boldsymbol{T}_{\boldsymbol{a}}\right)$ and thrust force vector $(\boldsymbol{f})$, given as

$$
\tau=T_{a} f .
$$

The thrust force vector becomes

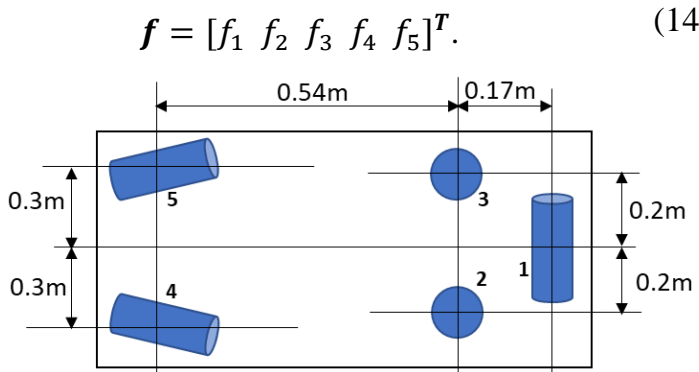

Figure 1: Position of thrusters on Minerva HAUV

The effect of each thruster on the vehicle is shown in the columns of the thrust allocation matrix. The thrust allocation matrix of Minerva HAUV is given as

$\boldsymbol{T}_{\boldsymbol{a}}=\left[\begin{array}{ccccc}0 & 0 & 0 & C \sigma & c \sigma \\ 1 & 0 & 0 & -s \sigma & s \sigma \\ 0 & 1 & 1 & 0 & 0 \\ 0 & l_{y 2} & l_{y 3} & 0 & 0 \\ 0 & 0 & 0 & 0 & 0 \\ l_{x 1} & 0 & 0 & l_{x 4} s \sigma+l_{y 4} c \sigma l_{x 5} s \sigma-l_{y 5} c \sigma\end{array}\right]$.

The position of each thruster with respect to $\mathrm{CO}$ is shown in Table 1.

Table 1: Position of thrusters on Minerva HAUV

\begin{tabular}{|l|c|c|c|}
\hline Thruster & $l_{x}(\mathbf{m})$ & $l_{y}(\mathbf{m})$ & $l_{z}(\mathbf{m})$ \\
\hline Thruster 1 & 0.17 & 0 & 0 \\
\hline Thruster 2 & 0 & 0.2 & 0 \\
\hline Thruster 3 & 0 & -0.2 & 0 \\
\hline Thruster 4 & -0.54 & 0.3 & 0 \\
\hline Thruster 5 & -0.54 & -0.3 & 0 \\
\hline
\end{tabular}

The axial thrusters, i.e. thruster 03 and 04, are installed at an angle $\sigma=10^{\circ}$ with the horizontal; therefore, they contribute towards both surge and sway motions.

In this paper, only the axial and transverse thrusters were considered as the vehicle was operating in the horizontal plane. Therefore, the thrust allocation matrix reduces to the following

$$
\boldsymbol{T}_{\boldsymbol{a}}=\left[\begin{array}{ccc}
0 & c \sigma & c \sigma \\
1 & -s \sigma & s \sigma \\
l_{x 1} & l_{x 4} s \sigma+l_{y 4} c \sigma & l_{x 5} s \sigma-l_{y 5} c \sigma
\end{array}\right] .
$$

\subsection{STATE-SPACE MODEL}

A state-space model of HAUV was also developed by linearising the equations of motion and including the disturbance and noise terms. This model is required to observe the effect of disturbance, noise and limited sensor readings on the motion response of the vehicle. Moreover, it is needed to decide on an appropriate filter to estimate the actual states of the vehicle and to design a control system to get the desired motion response under these limitations. In the horizontal plane, the state-space model is given as

$$
\begin{gathered}
\dot{x}=A x+B u+w(t) . \\
y=C x+v(t) .
\end{gathered}
$$

Where 


$$
\begin{aligned}
& \boldsymbol{A}=\left[\begin{array}{cccccc}
0 & 0 & 0 & 1 & 0 & 0 \\
0 & 0 & 0 & 0 & 1 & 0 \\
0 & 0 & 0 & 0 & 0 & 1 \\
0 & 0 & 0 & -\frac{X_{u}}{m+X_{\dot{u}}} & 0 & 0 \\
0 & 0 & 0 & 0 & -\frac{Y_{v}}{m+Y_{\dot{v}}} & 0 \\
0 & 0 & 0 & 0 & 0 & -\frac{N_{r}}{I_{z}+N_{\dot{r}}}
\end{array}\right] . \\
& \boldsymbol{B}=\left[\begin{array}{ccc}
0 & 0 & 0 \\
0 & 0 & 0 \\
0 & 0 & 0 \\
\frac{1}{m+X_{\dot{u}}} & 0 & 0 \\
0 & \frac{1}{m+Y_{\dot{v}}} & 0 \\
0 & 0 & \frac{1}{I_{z}+N_{\dot{r}}}
\end{array}\right] \\
& \boldsymbol{C}=\left[\begin{array}{llllll}
1 & 0 & 0 & 0 & 0 & 0 \\
0 & 1 & 0 & 0 & 0 & 0 \\
0 & 0 & 1 & 0 & 0 & 0
\end{array}\right] \text {. }
\end{aligned}
$$

$\boldsymbol{w}(\boldsymbol{t})$ and $\boldsymbol{v}(\boldsymbol{t})$ are included as Gaussian white noises for the disturbance and noise respectively (Graver, 2005).

\section{LF FORMATION CONTROL STRATEGY}

The LF formation control strategy ensures that the leader and follower vehicles maintain a predefined formation. This ensures the payload stability and avoids the internal collision between the vehicles and the payload.

The LF formation control strategy was developed for the underwater transportation system such that the leader and follower were controlled using PID controllers as these have very good performance for payload transportation (Anderlini, et al., 2018). Whereas a linear feedback controller was applied on the formation dynamics to get the desired pose for the follower. Three different methods were implemented to control the follower and following testing the best strategy was selected.

The pose of leader and follower and their relative terms are shown in Figure 2. The components of $\lambda$ in EFF are $x_{L}-$ $x_{F}$ and $y_{L}-y_{F}$. The subscripts $L$ and $F$ stand for leader and follower respectively. $\lambda$ can be transformed into BFF of the leader to get the distances with respect to the leader, given as

$$
\left[\begin{array}{l}
\lambda_{x} \\
\lambda_{y}
\end{array}\right]=\left[\begin{array}{cc}
-\cos \psi_{L} & -\sin \psi_{L} \\
\sin \psi_{L} & -\cos \psi_{L}
\end{array}\right]\left[\begin{array}{l}
x_{L}-x_{F} \\
y_{L}-y_{F}
\end{array}\right]
$$

Or

$$
\begin{gathered}
\lambda_{x}=-\left(x_{L}-x_{F}\right) \cos \psi_{L}-\left(y_{L}-y_{F}\right) \sin \psi_{L} . \\
\lambda_{y}=\left(x_{L}-x_{F}\right) \sin \psi_{L}-\left(y_{L}-y_{F}\right) \cos \psi_{L} .
\end{gathered}
$$

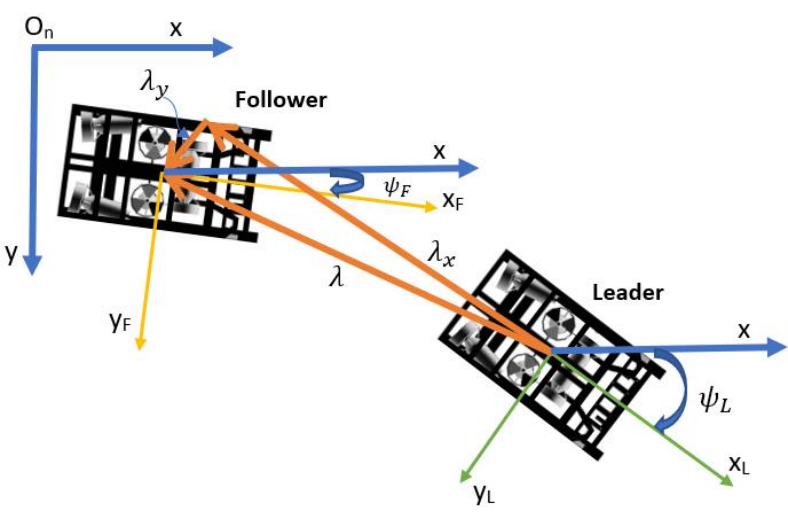

Figure 2: The pose of leader and follower and their relative terms

Differentiating equation (23) and (24) and using the kinematic equations (1) and (2) for transforming the velocity terms into BFF, the change of the distance between the leader and follower are obtained.

The formation dynamics are obtained by combining the change of the distance terms and angle between the leader and follower, which is written as

$$
\begin{gathered}
\dot{\lambda}_{x}=-u_{L}+u_{F} \cos \psi_{L F}+v_{F} \sin \psi_{L F}+\lambda_{y} r_{L}, \\
\dot{\lambda}_{y}=-v_{L}-u_{F} \sin \psi_{L F}+v_{F} \cos \psi_{L F}-\lambda_{x} r_{L}, \\
\dot{\psi}_{L F}=r_{L}-r_{F} .
\end{gathered}
$$

where $\left(u_{L}, v_{L}\right)$ and $\left(u_{F}, v_{F}\right)$ are the translational velocities of the leader and the follower respectively. Whereas, $r_{L}$ and $r_{F}$ are the angular velocities of the leader and the follower respectively. $\psi_{L F}$ is the difference between the leader and follower heading angles in EFF, given as

$$
\psi_{L F}=\psi_{L}-\psi_{F} .
$$

Putting equation (25) in the matrices form

$$
\dot{\chi}=\boldsymbol{F}(\chi)+\boldsymbol{G}(\chi) \boldsymbol{v},
$$

where

$$
\begin{aligned}
& \chi=\left[\begin{array}{lll}
\lambda_{x} & \lambda_{y} & \psi_{L F}
\end{array}\right]^{T}, \\
& \boldsymbol{v}=\left[\begin{array}{lll}
u_{F} & v_{F} & r_{F}
\end{array}\right]^{T} \text {, } \\
& \boldsymbol{F}(\chi)=\left[\begin{array}{c}
\lambda_{y} r_{L}-u_{L} \\
-\lambda_{x} r_{L}-v_{L} \\
r_{L}
\end{array}\right], \\
& \boldsymbol{G}(\chi)=\left[\begin{array}{ccc}
\cos \psi_{L F} & \sin \psi_{L F} & 0 \\
-\sin \psi_{L F} & \cos \psi_{L F} & 0 \\
0 & 0 & -1
\end{array}\right] \text {, }
\end{aligned}
$$

Using the dynamic inversion technique (Lau, 2014), the control input can be selected as

$$
\boldsymbol{v}=\boldsymbol{G}^{-\mathbf{1}}(\chi)(-\boldsymbol{F}(\chi)+\dot{\chi}) .
$$

Applying the linear feedback control law, we get

$$
\dot{\chi}=-\boldsymbol{k}\left(\chi-\chi_{d}\right) \text {. }
$$


As a result, the error between the desired and actual distances between the leader and follower $\left(e_{\lambda x}, e_{\lambda y}\right)$ and the heading angle difference between the leader and follower $\left(e_{\psi}\right)$ converges to zero asymptotically.

The developed formation control strategy is shown in Figure 3. A control system is first applied on the leader to get its desired pose. The pose of leader is communicated as input to the formation controller which is applied on $\left(e_{\lambda x}, e_{\lambda y}, e_{\psi}\right)$. The formation controller provides the desired translational and angular velocities for the follower $\left(u_{F d}, v_{F d}, r_{F d}\right)$ which are compared to the actual velocities $\left(u_{F d}, v_{F d}, r_{F d}\right)$ to get the error terms. The follower's control system is then applied on the error terms to get the desired motion response of the follower with respect to the leader. The control system for the leader and follower was designed using the PID controllers. The desired goal location was first set for the leader and the controllers were applied to reach it. The controllers are applied on the error between the desired and actual position of the leader. The output from the controllers, which are the control inputs for the system, are given as desired thrust vector $\left(\boldsymbol{\tau}_{\boldsymbol{d}}\right)$. This was multiplied by the inverse of the thrust allocation matrix $\left(\boldsymbol{T}_{\boldsymbol{a}}\right)$ to get the desired thrust force of the thrusters $\left(\boldsymbol{f}_{\boldsymbol{d}}\right)$. The saturation limit was then applied to get the actual thrust forces $(\boldsymbol{f})$.

The Minerva HAUV has a maximum thrust force of $340 \mathrm{~N}$ out the desired position and orientation of the follower which was then compared to the actual position and orientation of the follower to achieve the error terms on which the PID controllers were applied.

3. In Method 3, $\lambda_{x}$ and $\lambda_{y}$ were used to calculate the desired follower position, given as

$\left[\begin{array}{l}x_{F d} \\ y_{F d}\end{array}\right]=\left[\begin{array}{l}x_{L} \\ y_{L}\end{array}\right]-\left[\begin{array}{cc}-\cos \psi_{L} & -\sin \psi_{L} \\ \sin \psi_{L} & -\cos \psi_{L}\end{array}\right]^{-1}\left[\begin{array}{l}\lambda_{x} \\ \lambda_{y}\end{array}\right]$.

The PID controllers were then implemented on the difference between the desired and actual follower position and orientation to work out the control input for the system.

\section{TECHNICAL REQUIREMENTS}

The following technical requirements were set to be fulfilled for the success of the transportation mission:

1. The follower should follow the leader with a maximum tolerable Root Mean Square Error (RMSE) of $3 \mathrm{~m}$ to ensure the stability of payload and avoid internal collision between the vehicles and with the payload.

2. The leader should follow the trajectory with a maximum RMSE of $5 \mathrm{~m}$ for appropriate trajectory tracking which ensures the external collision

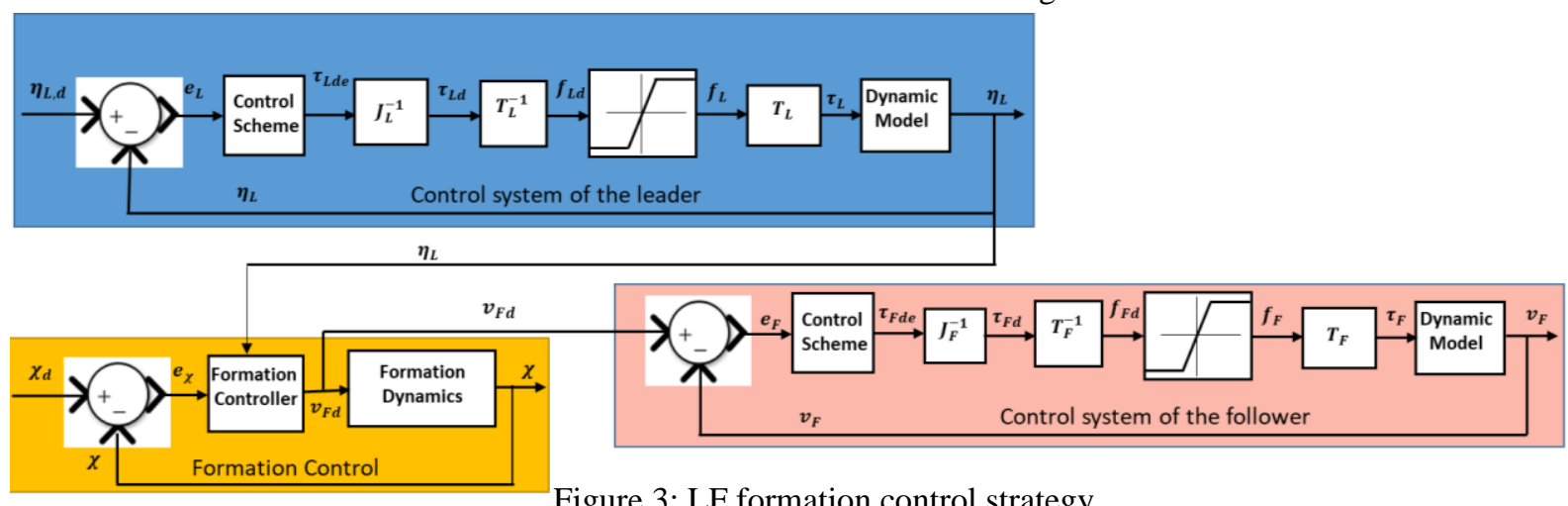

(Lysdahl, 2010) whereas the operating thrust force is $300 \mathrm{~N}$. The operating thrust force was taken as the thrust limit of the thruster to ensure the vehicles are operating at the efficient thrust ranges. Finally, $\boldsymbol{f}$ was multiplied by $\boldsymbol{T}_{\boldsymbol{a}}$ to get the actual thrust vector $(\boldsymbol{\tau})$. The difference between the control system of the leader and follower is that for the leader, the control system is applied on the error of the position and orientation of the vehicle and for the follower, on the error of the velocities.

The control system for the follower was implemented in the following three ways:

1. In Method 1, the desired follower velocities, which were obtained from the formation controller, were compared to the actual velocities of the follower to get the error terms. The PID controllers were then applied on those error terms.

2. In Method 2, the desired follower velocities, which were obtained from the formation controller, were given as input to the follower kinematics to work avoidance with the underwater obstacles.

3. The thrusters should be applied at the operating range i.e. $300 \mathrm{~N}$ which ensures fuel efficiency.

\section{SYSTEM DESCRIPTION}

The derived LF formation control strategy was implemented on the transportation system of two HAUVs which were connected to a payload via flexible links, as shown in Figure 4(a) (vertical plane) and Figure 4(b) (horizontal plane). This allows a margin of relative motion between the leader and follower which helps in the formation control. The payload of known weight was placed in a cubic container such that the total weight of the payload and container is equal to the buoyancy of container for neutral buoyancy. For the cable length of $3 \mathrm{~m}$ connected at the centre of the HAUV and the swing angle of $45^{\circ}$, the distance from the centre of each vehicle to the centre of the payload is $2.12 \mathrm{~m}$. This makes a total of $4.24 \mathrm{~m}$ between the centres of the leader and follower. It is considered that if the 
vehicles maintain a smooth formation throughout the motion, the payload remains stable.

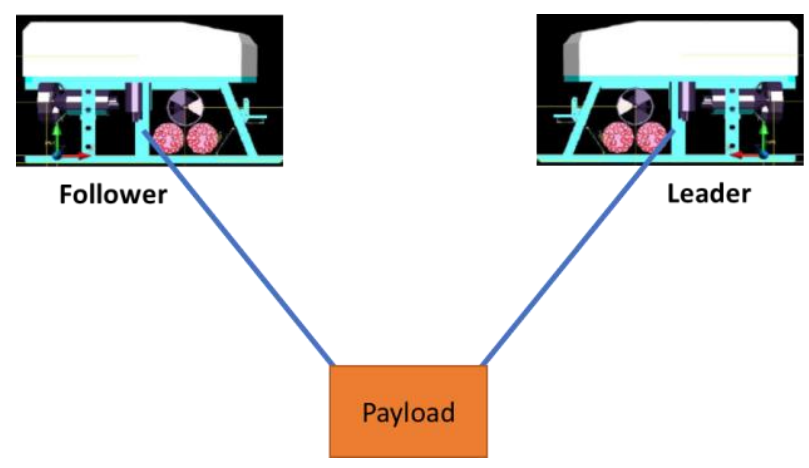

(a) Vertical plane

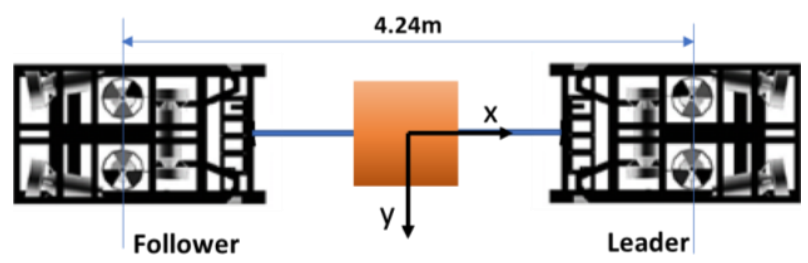

(b) Horizontal plane

Figure 4: Two Minerva HAUVs attached to a payload via flexible links

Both the vehicles were considered at zero initial position and orientation. Once the task starts, the follower moving to the desired distance and angle with respect to the leader ensures that both the leader and follower are getting to the desired position and orientation with respect to the payload.

The desired distance and angular difference between the leader and the follower are shown in Table 2.

Table 2: Desired distance and angular difference between the leader and follower

\begin{tabular}{|c|c|c|}
\hline$\lambda_{x}(m)$ & $\lambda_{y}(\mathrm{~m})$ & $\psi_{L F}($ degrees $)$ \\
\hline 4.24 & 0 & 0 \\
\hline
\end{tabular}

\section{TRAJECTORY GENERATION}

A trajectory is necessary between the start and goal location of the transportation system which is generated by avoiding the obstacles on the way. One way is to generate a curve trajectory for which the curve function is known (Yufka \& Ozkan, 2015)(Alothman, 2018)(Alothman, et al., 2017). Another way is to work out the waypoints based on path planning method and then develop a trajectory joining the waypoints. This method is mostly applied in practice as the waypoints are obtained avoiding the obstacles, including the safe margins to account for small deviation of the system from the desired trajectory. The path between two waypoints is called a segment. Therefore, if there are $n$ waypoints, the number of segments are $n-1$. The segment is the best possible route between the two adjacent waypoints which is obtained by defining an appropriate nominal function. The most implemented of such trajectories are minimum jerk trajectory and minimum snap trajectory (Mellinger \& Kumar, 2011; Salunkhe et al., 2016; Elmokadem et al., 2017; Rehman et al., 2019).

The curve trajectory depends on the time allocated for the overall trajectory. Whereas the waypoints trajectory depends on the time allocated between the waypoints.

Here a minimum trajectory was generated to test the efficiency of the developed LF formation control strategy in which the segment is defined by a seventh-order polynomial. The segment time is required to be optimised to complete the path between the waypoints at the minimum possible time while meeting the technical requirements and keeping the reasonable PID gains.

\section{RESULTS AND DISCUSSION}

Before providing the detail of the results, it is important to describe the hydrodynamic and hydrostatic parameters which were included in the simulation model of the system. The hydrodynamic parameters of the Minerva HAUV were acquired from reference (Mo, 2015), whereas, they were calculated for the cubic payload using the empirical approach (DNV, 2010).

As mentioned earlier that underwater communication systems have limitations which need the design of a sophisticated observer to estimate the position, orientation and velocity of the leader by the followers (Millan et al., 2014). However, these limitations were ignored.

Initially, the three methods of implementing the control system for the follower, as mentioned in Section 3, were tested.

The PID and formation control gains were selected such that the system reaches the goal location of $(30 \mathrm{~m}, 20 \mathrm{~m})$ in 200 secs starting from the origin. The formation controller gain in each direction was selected to be 0.5 . The chosen PID gains are shown in Table 3.

Table 3: PID gains for the leader and for the three methods for the follower

\begin{tabular}{|c|c|c|c|}
\hline & $K_{P}$ & $K_{I}$ & $K_{D}$ \\
\hline Leader & 2 & 0 & 0 \\
\hline Follower - Method 1 & 1 & 1 & 0 \\
\hline Follower -Method 2 & 5 & 0 & 5 \\
\hline Follower - Method 3 & 5 & 0 & 5 \\
\hline
\end{tabular}

For the leader, a proportional gain of 2 was enough to reach the goal location without significant overshoot and offset. In Method 1 for the follower, a small proportional and integral gains were sufficient to follow the leader, as shown in Figure 5(a) and (b). The follower moves to the desired axial distance in around 16 secs and then maintains that position throughout the simulation duration. Conversely, a small deviation is observed in sway. For Method 2 and 3, comparatively higher proportional gain was required to move the follower to the desired distance with respect to the leader and then follow it over the simulation duration. Moreover, a derivative gain of the same value as the proportional gain was essential to avoid the overshoot. Even then the follower took around 24 secs to reach the desired axial distance with respect to the leader. 


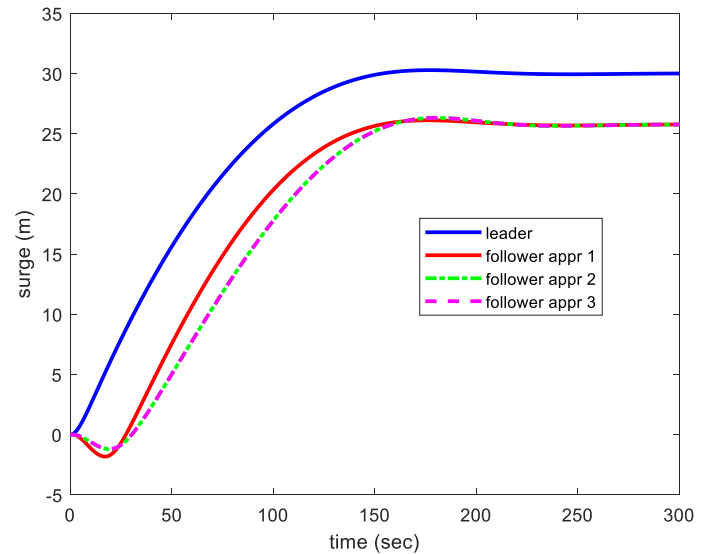

(a) Surge response

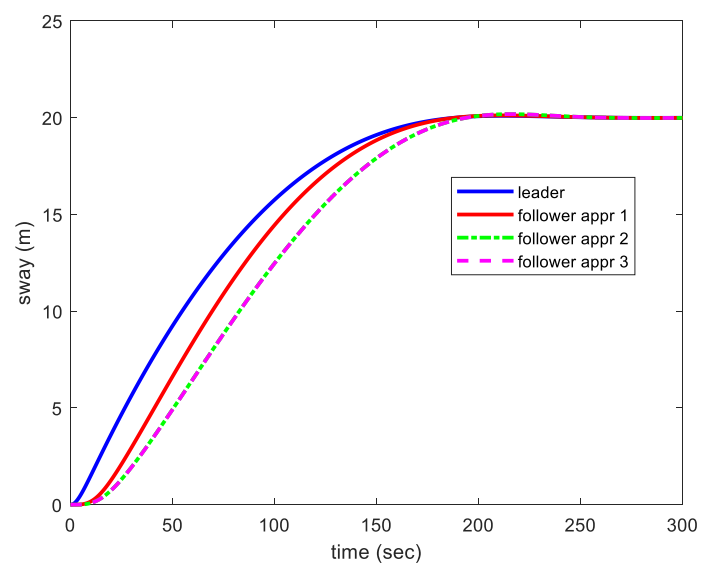

(b) Sway response

Figure 5: Motion response of the leader and the follower using the three methods for follower control

The RMSE of the follower's actual and desired position for the three methods are shown in Table 4. It can be seen that the RMSE in Method 1 is satisfying the first technical requirement in the axial direction but not in Method 2 and Method 3.

Table 4: RMSE of the followers for the three methods

\begin{tabular}{|c|c|c|}
\hline & $R M S E_{x}(m)$ & $R M S E_{y}(m)$ \\
\hline Method 1 & 1.20 & 0.69 \\
\hline Method 2 & 3.11 & 2.31 \\
\hline Method 3 & 3.12 & 2.30 \\
\hline
\end{tabular}

\subsection{TRAJECTORY TRACKING BY THE TRANSPORTATION SYSTEM}

The minimum snap trajectory was generated for the three waypoints, as shown in Table 5. This produced two trajectory segments. The trajectory tracking was tested at various segment times to achieve the optimised time under reasonable PID gains.

Table 5: Waypoints for the minimum snap trajectory

\begin{tabular}{|c|c|c|c|}
\hline & $x$ & $y$ & $\psi$ \\
\hline Waypoint 1 & 0 & 0 & 0 \\
\hline Waypoint 2 & 15 & 7 & 0 \\
\hline Waypoint 3 & 15 & 25 & 0 \\
\hline Waypoint 4 & 20 & 35 & 0 \\
\hline
\end{tabular}

Both the HAUVs were at the zero initial position when the leader was desired to follow the trajectory whereas the follower was controlled to first get to the desired distance with respect to the leader and then follow it accordingly.

Initial tests were performed at the higher segment times. For instance, at $100 \mathrm{secs}$, the trajectory was followed by the leader and the follower maintaining the formation fulfilling the RMSE's requirements for the leader and follower. Also, low PID and formation controller gains were sufficient to get the desired motion response. However, the time taken by the leader and the follower to complete the trajectory was very high and the thrusters were operating at very low thrust values which is not efficient.

The system was also tested at low segment times such as at 20secs. At moderate PID gains, higher time lags and higher RMSEs were experienced. Therefore, the technical requirements were not fulfilled. At higher Proportional gains between 200 and 400, the time lags were reduced but resulted in the overshoots for which higher derivative gains were required i.e. between 100 and 200. The higher derivative gains are not acceptable as they would magnify any noise in the signals. Moreover, a yaw moment was produced which resulted in undesirable heading angles and could result in the instability of the payload.

After several tests, the optimised segment time was selected to be 50secs. The PID gains at which the technical requirements were met are shown in Table 6. Moreover, the diagonal terms of the formation control gain matrix were kept at 1 . The differential gains are higher to avoid oscillations at the end of each segment.

\begin{tabular}{|c|c|c|c|c|}
\hline & & $K_{P}$ & $K_{I}$ & $K_{D}$ \\
\hline \multirow[t]{3}{*}{ Leader } & Surge & 10 & 0 & 30 \\
\hline & Sway & 20 & 0 & 30 \\
\hline & Yaw & 5 & 0 & 1 \\
\hline \multirow[t]{3}{*}{ Follower } & Surge & 5 & 3 & 10 \\
\hline & Sway & 5 & 5 & 10 \\
\hline & Yaw & 5 & 0 & 1 \\
\hline
\end{tabular}

Figure 6(a) shows the motion response of the system in the horizontal plane in which the leader HAUV is following the desired trajectory at a segment time of 50secs while follower HAUV is keeping the desired distance and angle with respect to the leader through out the trajectory. Figure 6(b) and (c) show the respective surge and sway response of the leader and follower HAUVs for better visualisation of their responses in each direction. The leader HAUV can be seen following the trajectory such that in surge, small time lags and overshoots are observed at the end of each segment. Whereas in sway, there is small time lags and offsets in each segment. To work out whether these variations are acceptable or not, RMSEs were worked out over the whole simulation duration. RMSEs of the leader's position from the reference trajectory, the follower's velocity from the desired velocity and the follower's position from the desired position were calculated, as shown in Table 7. All these variations are within the 
tolerable limits, as mentioned in the technical requirements in Section 4.

Table 7: RMSE of the leader and follower responses

\begin{tabular}{|c|c|c|}
\hline & $R M S E_{x}(m)$ & $R M S E_{y}(m)$ \\
\hline $\begin{array}{c}\text { Leader's position from } \\
\text { reference }\end{array}$ & 2.18 & 2.83 \\
\hline $\begin{array}{c}\text { Follower velocity from } \\
\text { desired velocity }\end{array}$ & 0.21 & 0.05 \\
\hline $\begin{array}{c}\text { Follower's position from } \\
\text { desired }\end{array}$ & 1.11 & 1.09 \\
\hline
\end{tabular}

Figure 6(d) shows the thrust forces and moment which are applied by the leader HAUV while following the desired trajectory. Due to operation in the horizontal plane, the thrust forces are applied in surge and sway while thrust moment is applied in yaw. It can be seen that the axial and the transverse thrusters apply a maximum thrust force of $66.5 \mathrm{~N}$ and $45 \mathrm{~N}$ respectively during the trajectory following in the first segment to move the vehicle towards waypoint 2 , as shown in Table 5. During the second segment, zero axial thrust force is applied as the desired surge distance is zero whereas a maximum sway thrust force of $154 \mathrm{~N}$ is applied to move the leader to $25 \mathrm{~m}$. During the third segment, a thrust force of $18 \mathrm{~N}$ and $73 \mathrm{~N}$ are applied in surge and sway respectively as only $5 \mathrm{~m}$ is moved in surge whereas $10 \mathrm{~m}$ in sway. The thrust forces by the leader are low but acceptable as they allow enough time for the follower to apply an initial axial thrust force of the maximum limit to keep following the leader.

Subsequently, the thrust forces and moment which are applied by the follower to keep track of the leader are shown in Figure 6(e). An initial axial thrust force of $-445 \mathrm{~N}$ is applied to move the follower to the desired axial distance with respect to the leader. Subsequently, a maximum thrust force of $63 \mathrm{~N}$ and $44 \mathrm{~N}$ are applied in surge and sway to follow the leader during the first segment. During the second segment, no axial thrust force is applied to maintain the axial position of the follower with respect to the leader, whereas, a maximum thrust force of $133.5 \mathrm{~N}$ is applied in sway to move the system to the desired sway distance of $25 \mathrm{~m}$. A thrust force of $20 \mathrm{~N}$ and $68 \mathrm{~N}$ are applied in surge and sway respectively to follow the leader during the third segment.

A small negative thrust force at the end of each segment is applied to slow down the system in order to keep track at the next segment.

No yaw moment is applied to avoid system turning and avoid causing stability problems for the payload.

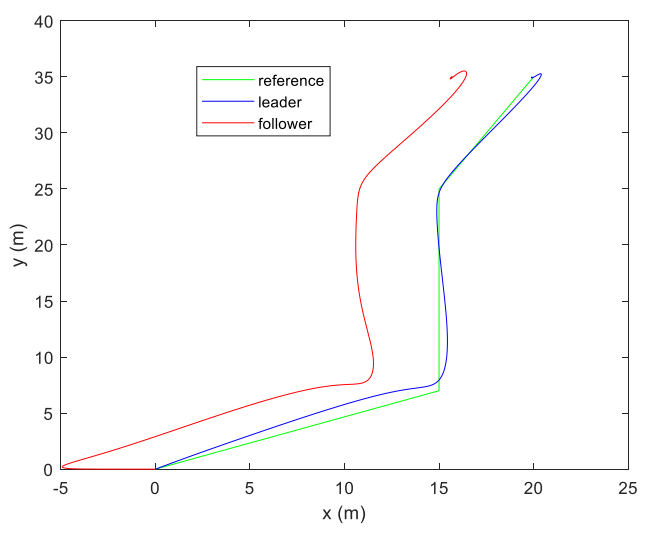

(a) Motion response in the horizontal plane

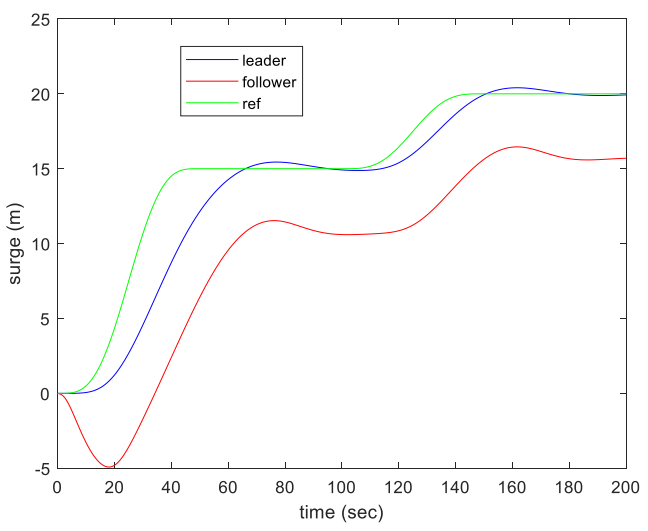

(b) Surge response

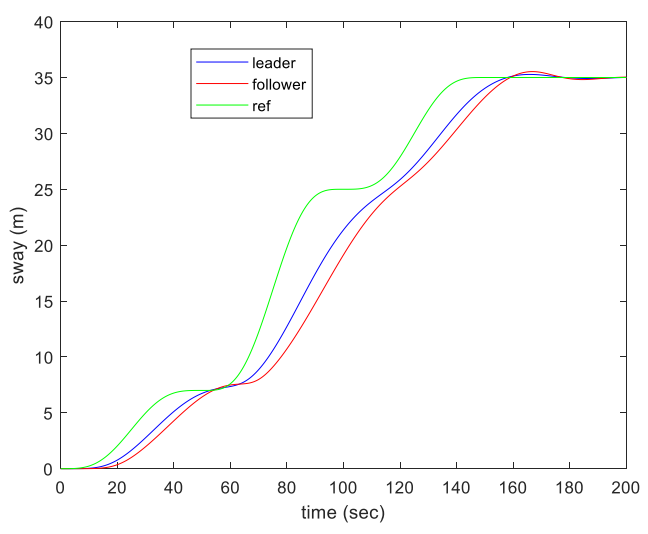

(c) Sway response
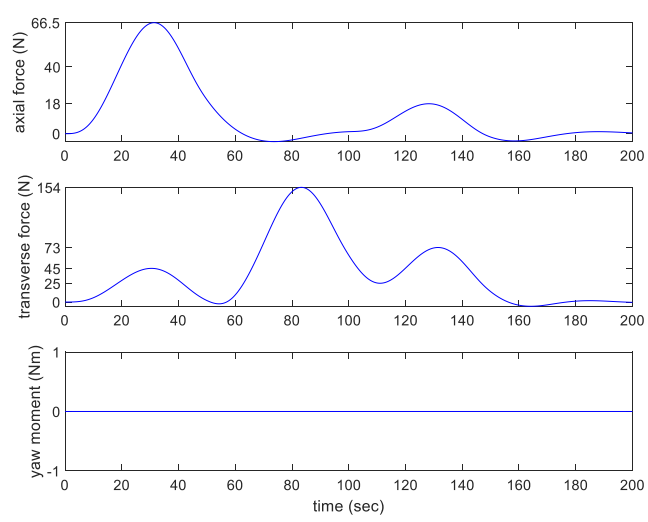

(d) Thrust response of the leader 

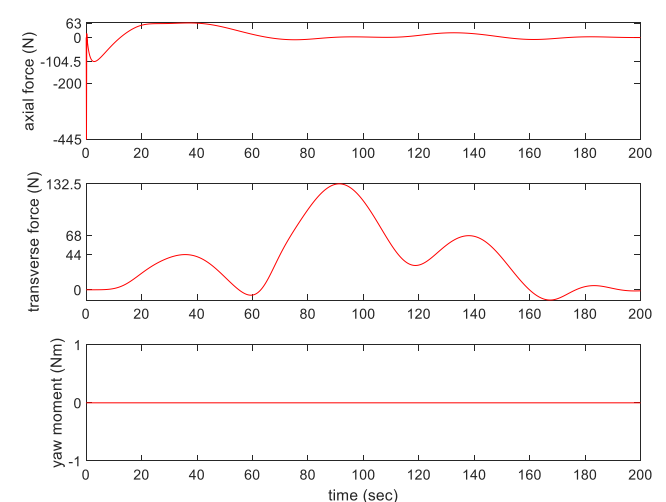

(e) Thrust response of the follower

Figure 6: System's response at the optimised segment time

\subsection{TRAJECTORY TRACKING UNDER DISTURBANCE, NOISE, SEA CURRENT AND LIMITED SENSOR DATA}

The randomly varying disturbance was added to each state of the leader. Moreover, it was considered that only pose of the leader could be measured by the sensors on the vehicle. The sensor readings are affected by random noise levels.

After confirming that the leader's model is controllable and observable, a Kalman Filter (KF) based on the Linear Quadratic Estimator (LQE) was developed to estimate the full state of the leader under disturbance, noise and limitation of sensor measurements. The LQE decides on the $\mathrm{KF}$ gains using the model parameters, disturbance and noise covariances. KF takes into consideration the model parameters, control inputs and $\mathrm{KF}$ gains to estimate the leader's states.

The reasonable ranges were chosen for the disturbance and noise, as shown in Table 8.

Table 8: Gaussian white noise for disturbance and noise

\begin{tabular}{|c|c|c|}
\hline & $\boldsymbol{w}(\boldsymbol{t})$ & $\boldsymbol{v}(\boldsymbol{t})$ \\
\hline Position & $\operatorname{rand}(0,2)$ & $\operatorname{rand}(0,1)$ \\
\hline Orientation & $\operatorname{rand}(0,2)$ & - \\
\hline Covariance & 0.1 & 1 \\
\hline
\end{tabular}

A test was performed to evaluate the KF performance. The leader was run for 50secs. Between 10 secs and 20secs, an axial thrust of $100 \mathrm{~N}$ was applied.

Figure 7 shows the surge response of leader. It can be seen that the estimated surge response by KF under signal noise and limited sensor readings is close to the actual surge response without noise and knowing the full state of the leader.

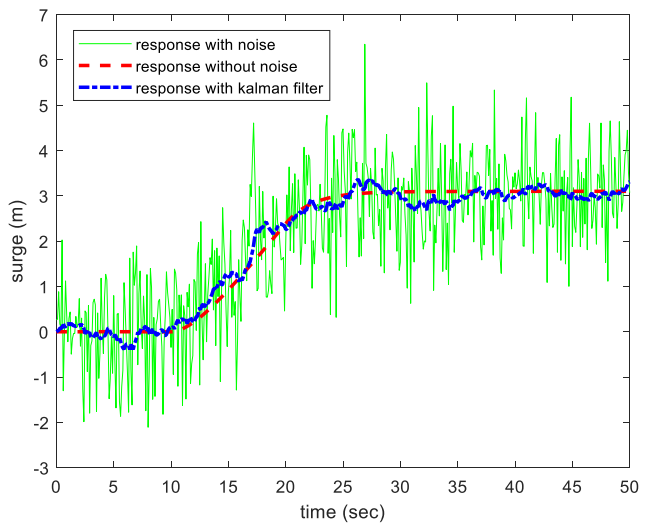

Figure 7: Surge response of the leader under disturbance and noise

A sea current at the constant velocity of $0.2 \mathrm{~m} / \mathrm{sec}$ was applied against the motion of the transportation system in surge. This is the maximum average sea current velocity which was measured underwater (Shanmugam, 2012). The equations of motion for both the leader and follower were modified by replacing $\boldsymbol{v}$ by $\boldsymbol{v}_{\boldsymbol{r}}$ in equation (4) and (11). Where $\boldsymbol{v}_{\boldsymbol{r}}=\boldsymbol{v}-\boldsymbol{v}_{\boldsymbol{c}}$. i.e. the difference between the velocity of the vehicle $(\boldsymbol{v})$ and the sea current velocity $\left(\boldsymbol{v}_{\boldsymbol{c}}\right)$.

After including the disturbance, noise and sea current under limited sensor readings, the leader was tasked to follow the same trajectory as mentioned in Section 7.1. The actual position, orientation and velocities of the leader were taken as estimated by the KF.

The formation control gains were kept at 1 . The reasonable PID gains at which the desired motion response was achieved are shown in Table 9.

Table 9: PID gains for the system experiencing disturbance, noise, sea current and limited sensor readings

\begin{tabular}{|c|c|c|c|c|}
\hline & & $K_{P}$ & $K_{I}$ & $K_{D}$ \\
\hline Leader & Surge & 20 & 0 & 30 \\
\cline { 2 - 5 } & Sway & 40 & 0 & 30 \\
\cline { 2 - 5 } & Yaw & 5 & 0 & 1 \\
\hline Follower & Surge & 5 & 2 & 10 \\
\cline { 2 - 5 } & Sway & 5 & 5.5 & 10 \\
\cline { 2 - 5 } & Yaw & 5 & 0 & 1 \\
\hline
\end{tabular}

The desired trajectory and its segment time for the leader HAUV are kept the same as used in Section 7.1 and the desired distance and angle of the follower with respect to the leader are kept the same as shown in Table 2. 

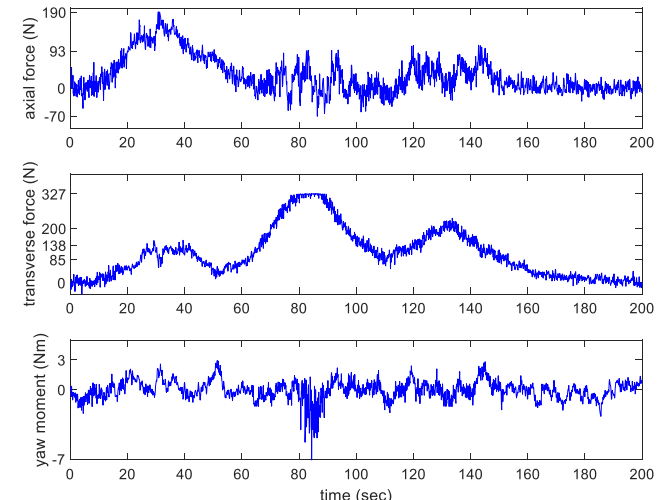

Leader's thrust response
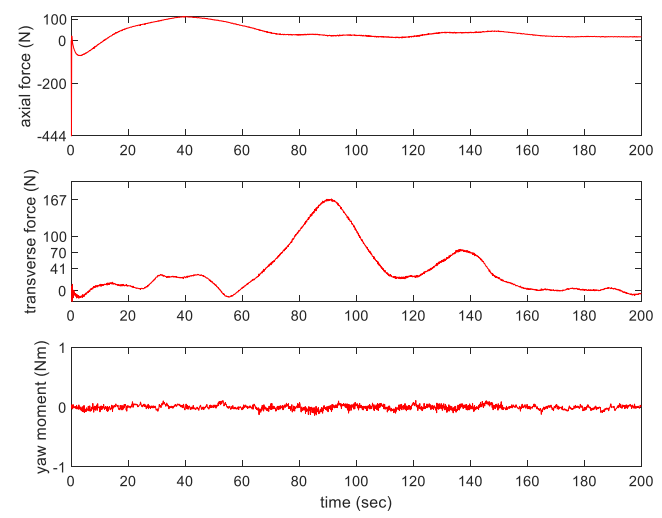

(a) Follower's thrust response

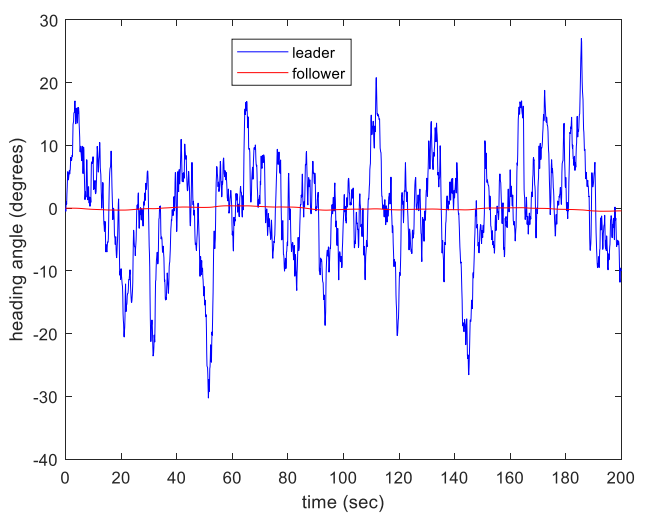

(b) Leader's and follower's heading angle response

Figure 8(a) shows the motion response of the leader and follower in the horizontal plane under the mentioned conditions. The respective surge and sway responses are shown in Figure 8(b) and Figure 8(c). It can be seen that the leader HAUV is following the trajectory with small oscillations whereas, the follower HAUV is maintaining the desired distance from leader throughout the trajectory without significant oscillations. The RMSEs of both the leader and follower are within the desired limits, as shown in Table 10.
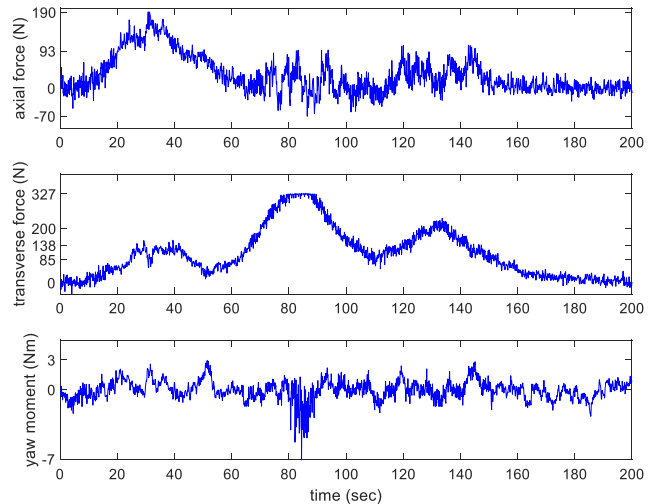

Leader's thrust response
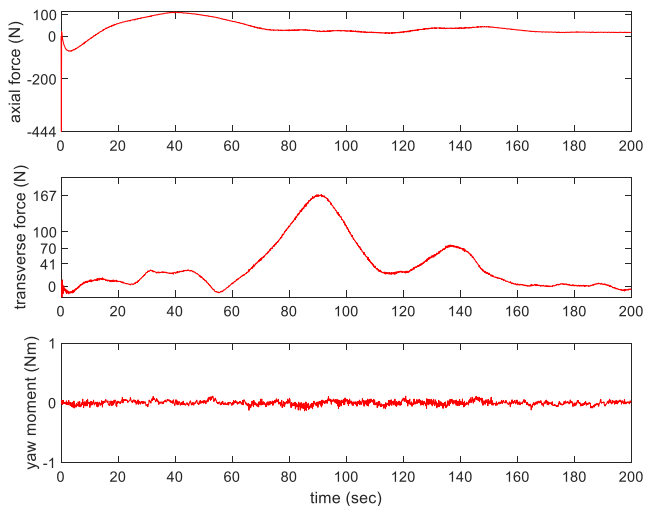

(c) Follower's thrust response

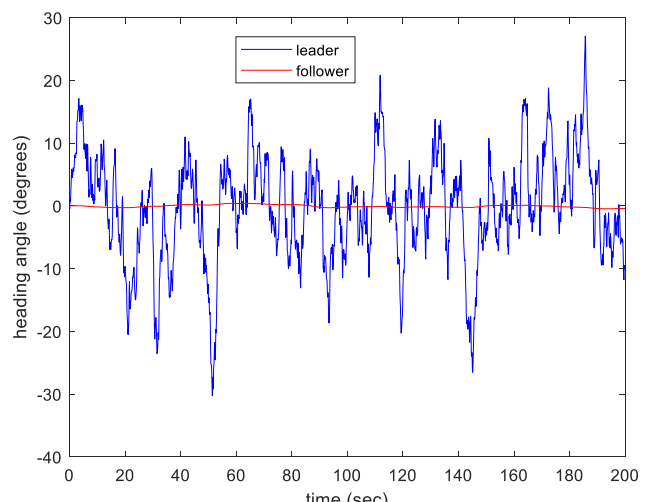

(d) Leader's and follower's heading angle response

Figure 8(d) shows the thrust forces and moment which are applied by the leader HAUV while following the desired trajectory under the mentioned conditions. The axial thrust force is applied at a maximum of $190 \mathrm{~N}$ during the first segment to move the leader to the axial distance of $15 \mathrm{~m}$. It then oscillates between $93 \mathrm{~N}$ and $-70 \mathrm{~N}$ during the second segment to maintain the axial position. Subsequently, the axial thrust force of $95 \mathrm{~N}$ is applied during the third segment to move the leader to the axial distance of $20 \mathrm{~m}$. On the other hand, the transverse thrust forces are applied at the maximum of $138 \mathrm{~N}, 327 \mathrm{~N}$ and $200 \mathrm{~N}$ during the first, second and third segments to move the vehicle to the transverse distance of $7 \mathrm{~m}, 25 \mathrm{~m}$ and $35 \mathrm{~m}$ respectively. This shows that the leader HAUV is operating at reasonable thrust limits and giving reasonable time to the follower to get the desired distance with respect to the leader. However, the thrust oscillations are observed due to the randomly varying 
disturbance and noise. The thrust moment oscillations can also be seen between $3 \mathrm{Nm}$ and $-7 \mathrm{Nm}$.

Figure 8(e) shows the thrust forces and moment which are applied by the follower to maintain the desired distance and angle with respect to the leader throughout the trajectory under the mentioned conditions. An axial thrust force of $444 \mathrm{~N}$ is initially applied to move the follower to the desired axial distance of $4.24 \mathrm{~m}$ with respect to the leader. It is then applied at a maximum of $100 \mathrm{~N}$ during the first segment and at small force values during the second and third segments to keep track of the leader in the axial direction. On the other hand, the transverse thrust forces of $41 \mathrm{~N}, 167 \mathrm{~N}$ and $70 \mathrm{~N}$ and by the follower are applied at the operating ranges without significant oscillations. Also, the yaw moment oscillations are negligible.

Figure 8(f) shows the heading angle response of the leader and follower HAUVs. The leader HAUV has high oscillations in heading angle due to the oscillatory yaw thrust moments. Whereas, the follower's heading angle is kept close to zero throughout motion due to negligible yaw moment oscillations.

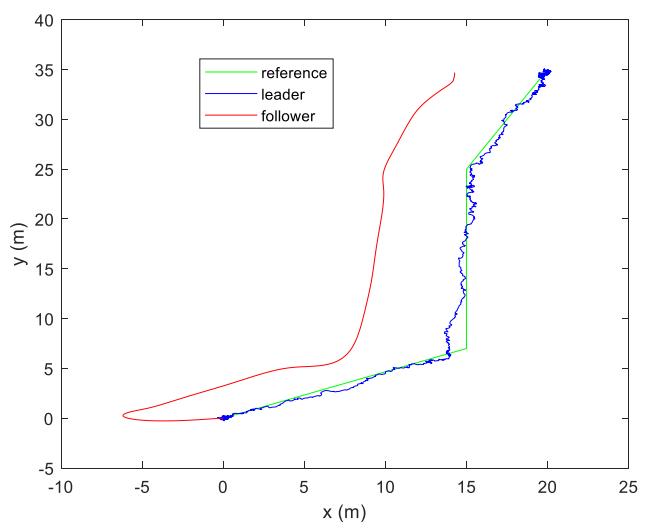

(e) Motion response in the horizontal plane

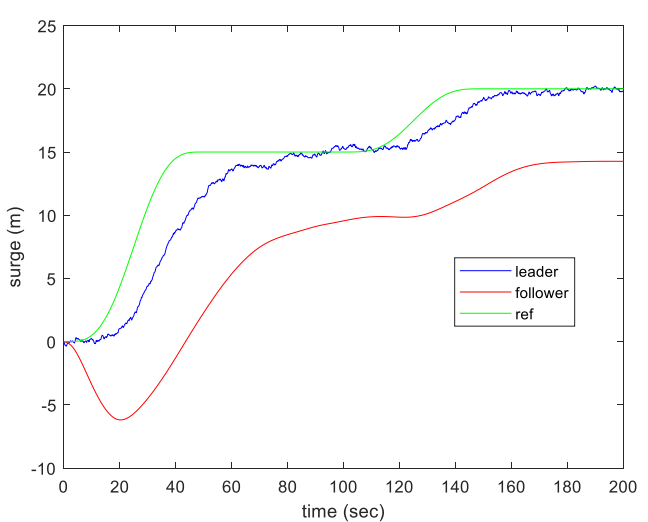

(f) Surge response

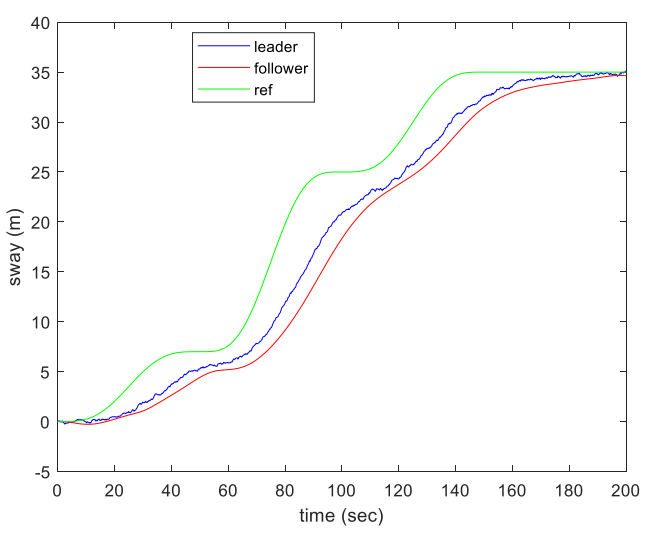

(g) Sway response
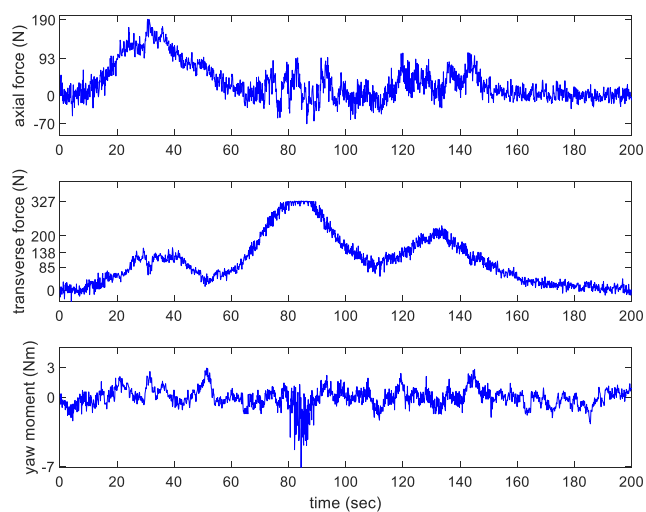

(h) Leader's thrust response
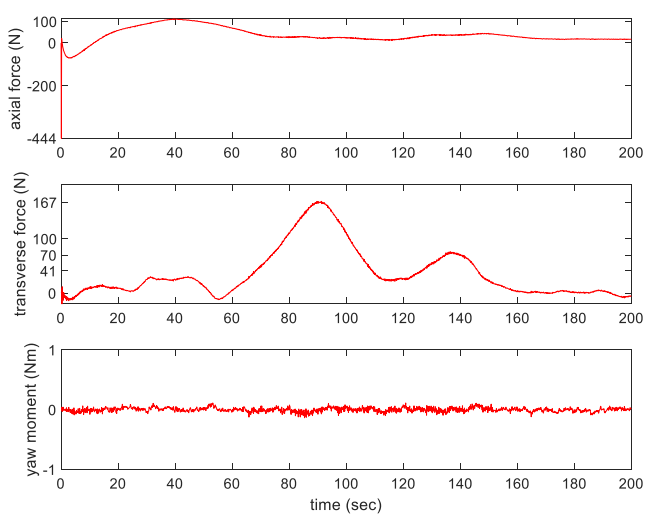

(i) Follower's thrust response

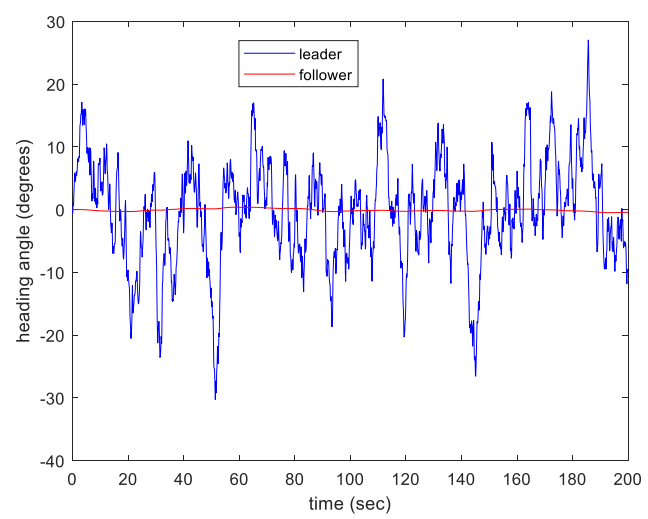

(j) Leader's and follower's heading angle response

Figure 8: System's response under disturbance, noise, sea current and limited sensor readings 
Table 10: RMSEs of the leader and follower under disturbance, noise, sea current and limited sensor readings

\begin{tabular}{|c|c|c|}
\hline $\begin{array}{c}\text { Leader's position from } \\
\text { reference }\end{array}$ & $R M S E_{x}(\mathrm{~m})$ & $R M S E_{y}(\mathrm{~m})$ \\
\hline $\begin{array}{c}\text { Follower's position from } \\
\text { desired }\end{array}$ & 2.79 & 3.58 \\
\hline
\end{tabular}

\section{CONCLUSIONS}

In this paper, a LF formation control strategy was developed for underwater transportation using the PID controllers for the HAUVs and the linear feedback controller for the formation. Initial optimisation was carried out to select the trajectory segment time at which the leader and follower fulfilled the set technical requirements in the minimum possible time and under reasonable PID gains. To test the robustness of the strategy, disturbance, measurement noise and sea current were included in the simulation under limited sensor readings. A Kalman filter was used to estimate the full state of the leader under the constraints, showing good accuracy. The motion response of the transportation system was meeting the desired RMSE's limits keeping the reasonable PID gains. However, the thrust oscillations were prominent which also caused undesired heading angles. One of the solutions is to introduce an average moving filter of the first order to the leader's states and then adjust the controller gains to meet the desired requirements. Moreover, to avoid high derivative gains and ease of selecting the controller gains for each state, the optimal controllers could be used for the leader and follower such as the Linear Quadratic Regulator (LQR).

The developed LF strategy is important for multi-vehicular underwater transportation as it ensures that the vehicles remain in the loop throughout the transportation task with stability regardless of the way they are connected to the payload. It will also avoid internal collisions between the vehicles and with the payload. Moreover, the external collisions with the underwater obstacles will also be avoided as the trajectory tracking is appropriate.

In the future, the non-dimensional parameters could be used so that they can be used for any type of autonomous underwater vehicle. Moreover, the underwater communication constraints could be included and Kalman Filter (KF) implemented on the follower to estimate the full state of the leader.

\section{REFERENCES}

1. AlOthMAN, Y. (2018) 'Using Constrained Model Predictive Control To Control Two Quadrotors Transporting A Cable-Suspended Payload', In 13th World Congress On Intelligent Control And Automation. IEEE, Pp. 228-233. DOI:10.1109/WCICA.2018.8630488.

2. ALOTHMAN, Y., GUO, M. AND GU, D. (2017) 'Using Iterative LQR To Control Two Quadrotors Transporting A Cable-Suspended Load', IFAC-
Papersonline, 50(1), Pp.

4324-4329.

DOI:10.1016/J.Ifacol.2017.08.861.

3. ANDERLINI, E., PARKER, G. G. AND THOMAS, G. (2018) 'Control Of A ROV Carrying An Object', Ocean Engineering, 165(March), Pp. 307-318. DOI:10.1016/J.Oceaneng.2018.07.022.

4. BEGGS, J. S. (1983) Kinematics. Taylors \& Francis.

5. BLIDBERG, D. R. (2010) 'The Development Of Autonomous Underwater Vehicles (AUV); A Brief Summary', IEEE ICRA, 6500, P. 12.

6. DAI, Y. ET AL. (2010) 'Leader-Follower Formation Control Of Multi-Robots By Using A Stable Tracking Control Methods', Advances In Swarm Intelligence, 248, Pp. 291-298. DOI:10.1007/978-3-642-134982_38.

7. DNV (2010) 'Modelling And Analysis Of Marine Operations', Tech. Rep. DNV-RP-H103, Pp. 1-91.

8. EIDSVIK, O. A. (2015) Identification Of Hydrodynamic Parameters For Remotely Operated Vehicles. Norwegian University Of Science And Technology.

9. ELMOKADEM, T., ZRIBI, M. AND YOUCEFTOUMI, K. (2017) 'Terminal Sliding Mode Control For The Trajectory Tracking Of Underactuated Autonomous Underwater Vehicles', Ocean Engineering, 129, Pp. 613-625. DOI:10.1016/J.Oceaneng.2016.10.032.

10. FARR, N. (2018) 'Underwater Acoustic/Optical Communications And Data Connectivity', Woods Hole Oceanographic Institution (WHOI).

11. FOSSEN, T. I. (2011) Handbook Of Marine Craft Hydrodynamics And Motion Control. 1st Ed. Sussex: John Wiley \& Sons.

12. FROST, A. R. And Mcmaster, A. P. (1996) 'The Development Of A Remotely Operated Vehicle ( ROV) For Aquaculture', (6), Pp. 461-483.

13. GHOMMAM, J. ET AL. (2010) 'Formation Path Following Control Of Unicycle-Type Mobile Robots', Robotics And Autonomous Systems, 58(5), Pp. $727-$ 736.

14. GRAVER, J. G. (2005) Underwater Gliders: Dynamics, Control And Design. Princeton University.

15. LAU, S. Y. (2014) Tensegrity-Based Formation Control Algorithms For Unmanned Vehicles. Queen's University Belfast.

16. LAWTON, J. R. T. ET AL. (2003) 'A Decentralized Approach To Formation Maneuvers', IEEE Transactions On Robotics And Automation, 19(6), Pp. 933-941.

17. LLORET, J. ET AL. (2012) 'Underwater Wireless 
Sensor Communications In The 2.4 Ghz ISM Frequency Band', Sensors (Basel), 12(4), Pp. 42374264. Available

At: Https://Www.Ncbi.Nlm.Nih.Gov/Pmc/Articles/PMC3 355409/.

18. LYSDAHL, C. (2010) Design Of Dynamic Positioning System For ROV Minerva. Master Thesis, Norwegian University Of Sceince And Technology.

19. MEHRJERDI, H., GHOMMAM, J. AND SAAD, M. (2011) 'Nonlinear Coordination Control For A Group Of Mobile Robots Using A Virtual Structure', Mechatronics, 21(7), Pp. 1147-1155. DOI:10.1016/J.Mechatronics.2011.06.006.

20. MELLINGER, D. AND KUMAR, V. (2011) 'Minimum Snap Trajectory Generation And Control For Quadrotors', Pp. 2520-2525.

21. MERCADO-RAVELL, D. A. ET AL. (2013) 'Quadrotors Flight Formation Control Using A Leader-Follower Approach', European Control Conference (ECC), (July), Pp. 3858-3863. DOI:10.23919/ECC.2013.6669637.

22. MILLAN, P. ET AL. (2014) 'Formation Control Of Autonomous Underwater Vehicles Subject To Communication Delays', IEEE Transactions On Control Systems Technology, 22(2), Pp. 770-777. DOI:10.1109/TCST.2013.2262768.

23. MILLER, T. F., GANDHI, F. S. AND RU, R. J. (2014) 'Morphing Hull Concept For Underwater Vehicles', 92, Pp. 92-102. DOI:10.1016/J.Oceaneng.2014.09.023.

24. MO, S. M. (2015) Development Of A Simulation Platform For ROV Systems, NTNU - Trondheim. Marine Technology Master Thesis, Norwegian University Of Science And Technology.

25. OGREN, P. AND EGERSTEDT, M. (2002) 'A Control Lyapunov Function Approach To Multiagent Coordination', IEEE Transactions On Robotics And Automation, 18(5), Pp. 847-851. DOI:10.1109/TRA.2002.804500.

26. PERAIRE, J. AND WIDNALL, J. (2008) 'Lecture L26 - 3D Rigid Body Dynamics : The Inertia Tensor', In Dynamics.

27. QI, X. (2014) 'Adaptive Coordinated Tracking Control Of Multiple Autonomous Underwater Vehicles', Ocean Engineering, $\quad 91, \quad$ Pp. 84-90. DOI:10.1016/J.Oceaneng.2014.08.019.

28. REHMAN, F. U., THOMAS, G. AND ANDERLINI, E. (2019) 'Centralized Control System Design For Underwater Transportation Using Two Hovering Autonomous Underwater Vehicles (Hauvs)', In IFAC-
Papersonline. Elsevier Ltd, Pp. 13-18. DOI:10.1016/J.Ifacol.2019.09.111.

29. REHMAN, F. U., THOMAS, G. AND ANDERLINI, E. (2019) 'Development Of A Simulation Platform For Underwater Transportation Using Two Hovering Autonomous Underwater Vehicles (Hauvs)', In Proceedings of The 6th International Conference Of Control, Dynamic Systems, And Robotics (CDSR'19). Ottawa, Pp. 1-8. DOI:10.11159/Cdsr19.138.

30. SALUNKHE, D. H. ET AL. (2016) 'Design, Trajectory Generation And Control of Quadrotor Research Platform', In International Conference On Robotics And Automation For Humanitarian Applications. Kollam: $\quad$ IEEE, $\quad$ Pp. 1-7. DOI:10.1109/RAHA.2016.7931876.

31. SHANMUGAM, G. (2012) 'New Perspectives On Deep-Water Sandstones', In Handbook Of Petroleum Exploration And Production.

32. WANG, W. H. ET AL. (2009) 'Design Of Low-Cost Unmanned Underwater Vehicle For Shallow Waters', International Journal Of Advanced Mechatronic Systems, 1(3), Pp. 194-202. DOI:10.1504/Ijamechs.2009.023202.

33. WANG, Y., YAN, WEISHENG AND YAN, WEI (2009) 'A Leader-Follower Formation Control Strategy For Auvs Based On Line-Of-Sight Guidance', 2009 International Conference On Mechatronics And Automation, (I), Pp. 4863-4867. DOI:10.1109/ICMA.2009.5246389.

34. WERNLI, R. (2000) Low Cost UUV's For Military Applications: Is The Technology Ready?, Space And Naval Warfare Systems Center. San Diego.

35. WILLIAMS, C. D. (2004) 'AUV Systems Research At The NRC-IOT: An Update', International Symposium On Underwater Technology, Pp. 59-73.

36. WU, F., CHEN, J. AND LIANG, Y. (2017) 'LeaderFollower Formation Control For Quadrotors', In IOP Conference Series: Materials Science And Engineering. Beijing. DOI:10.1088/1757899X/187/1/012016.

37. YANG, X. ET AL. (2004) 'A Decentralized Control System For Cooperative Transportation By Multiple Non-Holonomic Mobile Robots', International Journal Of Control, 77(10), Pp. 949-963. DOI:10.1080/00207170410001719765.

38. YU, Y. ET AL. (2017) 'Obstacle Avoidance Behavior Of Swarm Robots Based On Aggregation And Disaggregation Method', Simulation, 93(11), Pp. 885898. DOI:10.1177/0037549717711281.

39. YUFKA, A. AND OZKAN, M. (2015) 'Formation- 
Based Control Scheme For Cooperative

Transportation By Multiple Mobile Robots',

International Journal Of Advanced Robotic Systems,

12(9). DOI:10.5772/60972. 